\title{
Coeficientes de cultura da cenoura nas condições edafoclimáticas do Alto Paranaíba, no Estado de Minas Gerais ${ }^{1}$
}

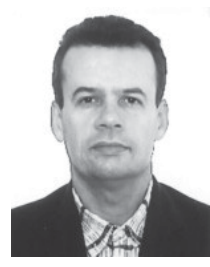

Rubens A. Oliveira ${ }^{2}$, Ismael de B. Rocha ${ }^{3}$, Gilberto C. Sediyama ${ }^{4}$, Mário Puiatti ${ }^{4}$, Paulo R. Cecon ${ }^{4} \&$ Suely de F. R. Silveira ${ }^{4}$

\author{
${ }^{1}$ Projeto financiado pela FAPEMIG \\ 2 DEA/UFV. CEP 36571-000, Viçosa, MG. Fone: (31) 3899-1909. E-mail: rubens@ufv.br (Foto) \\ ${ }^{3}$ Engenheiro Agrícola, Doutorando, UFV. E-mail: ismaelbr@vicosa.ufv.br \\ 4DEA/UFV. E-mail: sediyama@ufv.br, mpuiatti@ufv.br, cecon@dpi.ufv.br e sramos@ufv.br
}

Protocolo $94-9 / 7 / 2002$ - Aprovado em24/4/2003

\begin{abstract}
Resumo: A quantidade de água requerida em cada irrigação e o momento de sua aplicação, dependem das características físico-hídricas do solo e das condições climáticas locais e da cultura, considerando-se o seu estádio de desenvolvimento e a configuração de plantio. Neste trabalho, obtiveram-se coeficientes de cultura $\left(\mathrm{K}_{\mathrm{c}}\right)$ da cenoura, variedade Nantes, explorada nas condições edafoclimáticas da região do Alto Paranaíba, no Estado de Minas Gerais, seguindose a metodologia proposta no Boletim FAO 56, objetivando-se o manejo racional da água em áreas irrigadas por pivô central. Os valores de $\mathrm{K}_{\mathrm{c}}$ foram 1,15, 1,12, 1,12 e 1,10 para os estádios de desenvolvimento inicial, crescimento da cultura, intermediário e final, respectivamente. Nos estádios inicial e de crescimento, os valores de $\mathrm{K}_{\mathrm{c}}$ foram expressivamente maiores que aqueles obtidos com a metodologia FAO 24.
\end{abstract}

Palavras-chave: Daucus carota L., evapotranspiração, manejo de irrigação

\section{Crop coefficients of carrot for the 'Alto Parnaíba' region in the Minas Gerais State, Brazil}

\begin{abstract}
The amount of water required in each irrigation and the moment of its application depends on crop, soil type and the local climatic conditions. In this study crop coefficients $\left(\mathrm{K}_{\mathrm{c}}\right)$ were adjusted with the methodology proposed by FAO Irrigation and Drainage Paper 56, for irrigation scheduling of a carrot in the region of 'Alto Paranaíba', in the State of Minas Gerais, Brazil. The $\mathrm{K}_{\mathrm{c}}$ values for carrot grown in that region, irrigated by center pivot system, were 1.15, $1.12,1.12$ and 1.10 for beginning, initial, middle and final growth stages, respectively. In the beginning, initial and final growth stages, the obtained values of $\mathrm{K}_{\mathrm{c}}$ were larger than those obtained by the methodology proposed in the FAO Irrigation and Drainage Paper 24.
\end{abstract}

Key words: Daucus carota L., evapotranspiration, irrigation management

\section{INTRODUÇÃO}

A cenoura (Daucus carota L.) é uma hortaliça da família Apiácea, do grupo das raízes tuberosas, cultivada em larga escala nas regiões Sudeste e Sul do Brasil. Os maiores produtores são os municípios de Carandaí, Maria da Fé, São Gotardo e Rio Paranaíba, MG/ Piedade, Ibiúna e Mogi das Cruzes, SP/ Ponta Grossa, PR, e Irecê, BA.

As hortaliças são espécies vegetais de ciclo curto, sendo seu desenvolvimento intensamente influenciado pelas condições de umidade do ambiente (Silva \& Marouelli, 1998).

Em agricultura irrigada, o manejo da água deve ser adequado para cada espécie vegetal, sendo importante se conhecer alguns parâmetros básicos a respeito da necessidade de água das plantas. A determinação da evapotranspiração da cultura $\left(\mathrm{ET}_{\mathrm{c}}\right)$ requer o conhecimento da magnitude dos elementos meteorológicos e de vários parâmetros caracterizadores da superfície evaporante, que permitam estimar-se as resistências de superfície e aerodinâmica e os fluxos de calor sensível e latente.

$\mathrm{O}$ coeficiente de cultura $\left(\mathrm{K}_{\mathrm{c}}\right)$ representa a integração dos efeitos de três características que distinguem a $\mathrm{ET}_{\mathrm{c}}$ da evapotranspiração de referência (ETo): a altura da cultura, a resistência de superfície e o albedo da superfície cultura-solo. Durante o período vegetativo, o valor de $\mathrm{K}_{\mathrm{c}}$ varia com o desenvolvimento da cultura e com a fração de cobertura da superfície do solo, pela vegetação.

Doorenbos \& Pruitt (1977) apresentaram coeficientes de cultura para várias espécies de interesse agronômico e recomendaram que sejam realizados estudos regionais visando ajustarse coeficientes de cultura para as condições edafoclimáticas locais e as características varietais. 
Aragão Júnior (1983) obteve, em experimento conduzido em Guaramiranga, $\mathrm{CE}$, valores de $\mathrm{K}_{\mathrm{c}}$ para a cultura da cenoura, variedade Brasília, iguais a 0,77, 0,99 e 0,86, para os três últimos estádios de desenvolvimento. Carvalho (1994) encontrou, em experimento conduzido em Viçosa, MG, com a mesma variedade, os seguintes valores de $\mathrm{K}_{\mathrm{c}}$ para os quatro estádios de desenvolvimento: 1,03, 0,80, 1,16 e 1,61. Em experimento conduzido em lisímetros com lençol freático constante, em Viçosa, MG, Giacoia Neto (1996) obteve os seguintes valores de $\mathrm{K}_{\mathrm{c}}$ também para essa variedade, com profundidade do lençol freático mantida em $35 \mathrm{~cm}$ : 0,48, 0,77, 1,47 e 1,56. Silva \& Marouelli (1998) apresentam valores de $\mathrm{K}_{\mathrm{c}}$ para cenoura variando entre 0,70 e 0,90 no estádio inicial, com irrigações diárias, entre 0,70 e 0,85 no estádio de crescimento rápido; entre 1,00 e 1,15 no estádio intermediário, e entre 0,70 e 0,85 no estádio final. As diferenças entre os valores dos coeficientes de cultura devem-se, provavelmente, ao uso de métodos diferentes para se estimar a ETo. Carvalho (1994) usou a equação de Penman modificada pela FAO 24, enquanto Giacoia Neto (1996) utilizou o tanque Classe A, e Silva \& Marouelli (1998) adaptaram dados da FAO 24. A equação de Penman-Monteith é recomendada pela FAO como padrão de estimativa da evapotranspiração de referência (Pereira \& Allen, 1997).

Os coeficientes de cultura podem ser determinados de duas formas: os $\mathrm{K}_{\mathrm{c}}$ duplos e médios. Os $\mathrm{K}_{\mathrm{c}}$ duplos são obtidos pela expressão (Pereira \& Allen, 1997):

$$
\mathrm{K}_{\mathrm{c}}=\mathrm{K}_{\mathrm{s}} \mathrm{K}_{\mathrm{cb}}+\mathrm{K}_{\mathrm{e}}
$$

em que:

$\mathrm{K}_{\mathrm{s}}$ - coeficiente de déficit de umidade do solo, adimensional

$\mathrm{K}_{\mathrm{cb}}$ - coeficiente basal de cultura, adimensional

$\mathrm{K}_{\mathrm{e}}$ - coeficiente de evaporação na superfície do solo, adimensional

O coeficiente basal de cultura é a razão entre a $E_{c}$ e a $E_{0}$ quando a camada superficial do solo se encontra seca, mas tendo a umidade na zona radicular adequada para manter a demanda atmosférica. $\mathrm{O} \mathrm{K}_{\mathrm{cb}}$ representa o limite inferior do $\mathrm{K}_{\mathrm{c}}$ quando se subtraem os efeitos do umedecimento da camada superficial do solo pela irrigação ou precipitação. $\mathrm{O} \mathrm{K}_{\mathrm{s}}$ reduz o valor de $\mathrm{K}_{\mathrm{cb}}$ quando a umidade do solo na zona radicular é insuficiente para atender a plena transpiração das plantas e o $\mathrm{K}_{\mathrm{e}}$ representa a componente da evaporação da água presente na superfície do solo.

Os $\mathrm{K}_{\mathrm{c}}$ médios são expressos da seguinte forma:

$$
\mathrm{K}_{\mathrm{c}}=\overline{\mathrm{K}}_{\mathrm{cb}}+\overline{\mathrm{K}}_{\mathrm{e}}
$$

em que a soma $\overline{\mathrm{K}}_{\mathrm{cb}}+\overline{\mathrm{K}}_{\mathrm{e}}$ representa a média temporal dos efeitos conjugados da transpiração da cultura e da evaporação na superfície do solo, assumindo-se $\mathrm{K}_{\mathrm{s}}=1$.

Os $\mathrm{K}_{\mathrm{c}}$ médios correspondem àqueles divulgados em numerosas publicações, principalmente as da FAO. Os $\mathrm{K}_{\mathrm{c}}$ médios incluem efeitos gerais de umedecimento do solo pela irrigação ou pela precipitação pluvial e que são utilizados para se calcular a $\mathrm{ET}_{\mathrm{c}}$ relativa a períodos de vários dias, turno de rega maior, sobretudo para a condução da irrigação por gravidade ou aspersão convencional. Quando é necessário o conhecimento da $\mathrm{ET}_{\mathrm{c}}$ diária, utiliza-se o método dos $\mathrm{K}_{\mathrm{c}}$ duplos (Pereira \& Allen, 1997).

Objetivou-se, com este trabalho, obter os coeficientes de cultura para a cenoura, seguindo-se a metodologia proposta no Boletim FAO 56 (Allen et al., 1998) de maneira a permitir o manejo racional da água dessa cultura, explorada nas condições edafoclimáticas da região do Alto Paranaíba, no Estado de Minas Gerais e irrigada por pivô central.

\section{MATERIAL E MÉTODOS}

A pesquisa foi conduzida na Fazenda Experimental da Cooperativa Agropecuária do Alto Paranaíba (COOPADAP), localizada no município de Rio Paranaíba, $\mathrm{MG}$, com coordenadas geográficas de $19^{\circ} 12^{\prime} 26^{\prime}$ 'S de latitude, $46^{\circ} 09^{\prime} 46^{\prime \prime} \mathrm{W}$ de longitude e altitude de $1.159 \mathrm{~m}$ e clima Cwa, segundo classificação de Köeppen. Nessa cooperativa, a cenoura é cultivada durante todo o ano, com área plantada variando entre 4.000 e 5.000 ha.

O solo da área experimental foi classificado como Latossolo Vermelho-Amarelo, textura argilosa. A distribuição granulométrica e os resultados das análises físico-hídricas, encontram-se na Tabela $1 \mathrm{~A}$ e $\mathrm{B}$, tem-se o resultado da análise química do solo da área experimental.

Tabela 1. Distribuição granulométrica e resultado das análises físico-hídrica e química do solo da área experimental

\begin{tabular}{lccc} 
& \multirow{2}{*}{ Características } & Unidades & \multicolumn{2}{c}{ Profundidade - cm } \\
\cline { 3 - 4 } & & $0-20$ & $20-40$ \\
\hline A. Físico-hídrica & & & \\
Argila & $\mathrm{g} \mathrm{kg}^{-1}$ & 610 & 410 \\
Silte & $\mathrm{g} \mathrm{kg}^{-1}$ & 280 & 420 \\
Areia & $\mathrm{g} \mathrm{kg}^{-1}$ & 110 & 170 \\
Capacidade de campo & $\mathrm{g} \mathrm{kg}^{-1}$ & 320 & 310 \\
Ponto de murcha & $\mathrm{g} \mathrm{kg}^{-1}$ & 210 & 200 \\
Massa específica & $\mathrm{hg} \mathrm{dm}^{-3}$ & 1,04 & 1,03 \\
\hline B. Químicas & & & \\
pH em H ${ }_{2} \mathrm{O}$ & & 6,3 & 5,8 \\
Fósforo & $\mathrm{mg} \mathrm{dm}^{-3}$ & 4,4 & 0,8 \\
Potássio & $\mathrm{mg} \mathrm{dm}^{-3}$ & 36 & 18 \\
Cálcio & $\mathrm{cmolc} \mathrm{dm}^{-3}$ & 2,9 & 0,8 \\
Magnésio & $\mathrm{cmolc} \mathrm{dm}^{-3}$ & 0,7 & 0,1 \\
Alumínio & $\mathrm{cmolc} \mathrm{dm}^{-3}$ & 0,0 & 0,0 \\
Hidr. + Alumínio & $\mathrm{cmolc} \mathrm{dm}^{-3}$ & 6,1 & 5,9 \\
Matéria Orgânica & $\mathrm{dag} \mathrm{kg}^{-1}$ & 4,65 & 3,89 \\
\hline
\end{tabular}

O preparo do solo constou de uma aração e duas gradagens, seguidas de passagem da rotocanteiradora. $\mathrm{O}$ experimento foi conduzido em cinco canteiros de $1,75 \times 81,0 \mathrm{~m}$, plantados com cenoura (Daucus carota L.) da variedade Nantes, sendo o canteiro central utilizado para coleta dos dados.

O plantio foi feito em quatro linhas duplas espaçadas $0,40 \mathrm{~m}$, com sementes espaçadas $0,02 \mathrm{~m}$. Na área experimental foram aplicados, 15 dias antes do plantio, $180 \mathrm{~kg} \mathrm{ha}^{-1} \mathrm{de} \mathrm{N}$, na forma de sulfato de amônio, $220 \mathrm{~kg} \mathrm{ha}^{-1}$ de $\mathrm{K}_{2} \mathrm{O}$ na forma de 
cloreto de potássio, $2.222 \mathrm{~kg} \mathrm{ha}^{-1}$ de $\mathrm{P}_{2} \mathrm{O}_{5}$ na forma de superfosfato simples e $3 \mathrm{t} \mathrm{ha}^{-1}$ de calcário dolomítico, para elevar a saturação de bases, conforme recomendação da Comissão de Fertilidade do Solo do Estado de Minas Gerais (1999).

A emergência das plântulas ocorreu em 24 de maio de 2001, ou seja, 13 dias após a semeadura. Aos 20 e 40 dias após o plantio, aplicaram-se $210 \mathrm{~kg} \mathrm{ha}^{-1}$ de $\mathrm{N}$ e $166 \mathrm{~kg} \mathrm{ha}^{-1} \mathrm{de} \mathrm{K}_{2} \mathrm{O}$ em cobertura. $\mathrm{O}$ desbaste foi feito 35 dias após a emergência, adequando-se o espaçamento para 0,04 $\mathrm{m}$ entre plantas na fileira, estabelecendo-se uma população de 150 plantas $\mathrm{m}^{-2}$.

$\mathrm{Na}$ área experimental foi instalada uma estação meteorológica automática compacta, com sensores de medição da radiação solar, precipitação pluvial, velocidade do vento, temperatura e umidade relativa do ar. A coleta diária de dados climáticos foi feita às $8 \mathrm{~h}$.

A evapotranspiração de referência $\left(\mathrm{ET}_{\mathrm{o}}\right)$ foi estimada aplicando-se a equação de Penman-Monteith, em base diária (Allen et al., 1998). A metodologia utilizada no cálculo da ET considerou os efeitos de umedecimento da camada superficial do solo no valor do coeficiente de cultura, particionado em $\mathrm{K}_{\mathrm{cb}}$ e $\mathrm{K}_{\mathrm{e}}$. Assim, a $\mathrm{ET}_{\mathrm{c}}$ foi estimada de acordo com a seguinte equação:

$$
\mathrm{ET}_{\mathrm{c}}=\left(\mathrm{K}_{\mathrm{cb}}+\mathrm{K}_{\mathrm{e}}\right) \mathrm{ET}_{\mathrm{o}}
$$

O ciclo da cultura da cenoura foi dividido em quatro estádios, de acordo com os parâmetros propostos por Doorenbos \& Pruitt (1977) para a determinação dos $\mathrm{K}_{\mathrm{cb}}$ correspondentes: inicial - 20 dias; crescimento da cultura - 32 dias; intermediário - 50 dias; e final - 24 dias. A colheita das raízes de cenoura foi realizada aos 126 dias após o plantio.

$\mathrm{Na}$ determinação dos coeficientes basais de cultura foram usados os valores recomendados por Allen et al. (1998), para a condição sem estresse: $\mathrm{k}_{\mathrm{cb}}$ inicial $=0,15, \mathrm{k}_{\mathrm{cb}}$ intermediário $=0,95$ $\mathrm{e}_{\mathrm{cb}}$ final $=0,85$. $\mathrm{O}$ ajuste dos coeficientes basais de cultura, para os períodos intermediário e final, foi feito por meio da aplicação da seguinte equação:

$$
\mathrm{K}_{\mathrm{cb}}=\mathrm{K}_{\mathrm{cb}(\mathrm{tab})}+\left[0,04\left(\mathrm{u}_{2}-2\right)-0,004\left(\mathrm{UR}_{\min }-45\right)\right]\left(\frac{\mathrm{h}}{3}\right)^{0,3}
$$

em que:

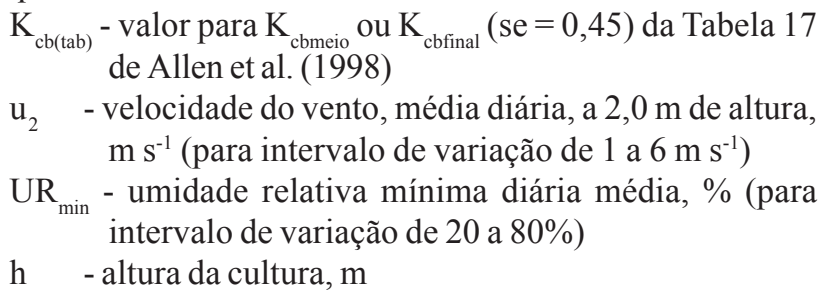

O coeficiente de evaporação foi calculado aplicando-se a equação:

$$
\mathrm{K}_{\mathrm{e}}=\min \left(\mathrm{K}_{\mathrm{r}}\left(\mathrm{K}_{\mathrm{c} \max }-\mathrm{K}_{\mathrm{cb}}\right), \mathrm{f}_{\mathrm{ew}} \mathrm{K}_{\mathrm{c} \max }\right)
$$

em que:

$\mathrm{K}_{\mathrm{r}}$ - coeficiente de redução da evaporação $\mathrm{f}_{\mathrm{ew}} \quad$ - fração do solo que está exposto e umedecido

$\mathrm{K}_{\mathrm{c} \max }$ - valor máximo de $\mathrm{K}_{\mathrm{c}}$ após chuva ou irrigação

Na determinação do $K_{\mathrm{r}}$, consideram-se duas fases: 1) logo após uma chuva ou irrigação, a evaporação na superfície umedecida do solo ocorre a uma taxa máxima e depende somente da energia disponível; assim, $\mathrm{K}_{\mathrm{r}}=1$; e, 2) a ocorrência de evaporação na superfície do solo limita a evaporação da água contida no perfil, adotando-se a profundidade de $0,15 \mathrm{~m}$; neste caso, $\mathrm{K}_{\mathrm{r}}$ é calculado por:

$$
\mathrm{K}_{\mathrm{r}}=\frac{\mathrm{TEW}-\mathrm{D}_{\mathrm{e}, \mathrm{i}-1}}{\mathrm{TEW}-\mathrm{REW}} \operatorname{para~}_{\mathrm{e}, \mathrm{i}-1}>\mathrm{REW}
$$

em que:

TEW - lâmina máxima de água que pode ser evaporada do solo, $\mathrm{mm}$

$\mathrm{D}_{\mathrm{e}, \mathrm{i}-1}$ - lâmina de evaporação acumulada na camada superficial do solo até o final do dia anterior, $\mathrm{mm}$

REW - lâmina de evaporação acumulada até o final da fase $1, \mathrm{~mm}$

A lâmina de água máxima que pode ser evaporada do solo foi calculada aplicando-se a seguinte equação:

$$
\mathrm{TEW}=1000\left(\theta_{\mathrm{CC}}-0,5 \theta_{\mathrm{PM}}\right) \mathrm{Z}_{\mathrm{e}}
$$

em que:

$\theta_{\mathrm{CC}}$ - umidade do solo na capacidade de campo, $\mathrm{m}^{3} \mathrm{~m}^{-3}$

$\theta_{\mathrm{PM}}$ - umidade do solo no ponto de murcha permanente, $\mathrm{m}^{3} \mathrm{~m}^{-3}$

$\mathrm{Z}_{\mathrm{e}} \quad$ - profundidade do solo sujeita a evaporação $(0,10 \mathrm{a}$ $0,15 \mathrm{~m})$. Assumiu-se $\mathrm{Z}_{\mathrm{e}}$ igual a $0,15 \mathrm{~m}$

O valor de $\mathrm{K}_{\mathrm{c} \max }$ foi obtido com a aplicação da equação:

$$
\begin{aligned}
\mathrm{K}_{\mathrm{c} \max }= & \max \left\{1,1+\left[0,04\left(\mathrm{u}_{2}-2\right)-\right.\right. \\
& \left.0,004(\mathrm{UR}-45)]\left(\frac{\mathrm{h}}{3}\right)^{0,3}, \mathrm{~K}_{\mathrm{cb}}+0,05\right\}
\end{aligned}
$$

A fração de solo exposta e umedecida $\left(\mathrm{f}_{\mathrm{ew}}\right)$, na qual ocorre a maior parte da evaporação, foi calculada por:

$$
\mathrm{f}_{\mathrm{ew}}=\min \left(1-\mathrm{f}_{\mathrm{c}}, \mathrm{f}_{\mathrm{w}}\right)
$$

em que:

1 - $\mathrm{f}_{\mathrm{c}}$ - fração média de solo exposto, não-sombreado $(0,01$ a 1)

$\mathrm{f}_{\mathrm{w}} \quad$ - fração média da superfície do solo molhada por irrigação ou chuva (0,01 a 1); no caso de irrigação por aspersão, $\mathrm{f}_{\mathrm{w}}=1$ (Allen et al., 1998) 
A fração efetiva da superfície do solo sombreada pela vegetação foi estimada pela equação seguinte:

$$
\mathrm{f}_{\mathrm{c}}=\left(\frac{\mathrm{K}_{\mathrm{cb}}-\mathrm{K}_{\mathrm{c} \text { min }}}{\mathrm{K}_{\mathrm{c} \text { max }}-\mathrm{K}_{\mathrm{c} \text { min }}}\right)^{(1+0,5 \mathrm{~h})}
$$

em que $\mathrm{K}_{\mathrm{c} \text { min }}$ é o menor valor de $\mathrm{K}_{\mathrm{c}}$ para solo com superfície descoberta e seca $(\approx 0,15$ a 0,20$)$.

Os cálculos foram efetuados com uso do programa REFET, para estimar a ETo pelo método Penman-Monteith, e da planilha FAO-56 (Allen et al., 1998) para estimar o K.

\section{RESULTADOS E DISCUSSÃO}

Os valores dos elementos climáticos precipitação, radiação solar, temperatura, umidade relativa e vento foram obtidos com uso de uma estação meteorológica automática, apresentados nas Figuras de 1 e 2, respectivamente. As lâminas de irrigação aplicadas durante o período experimental estão na Figura 1.

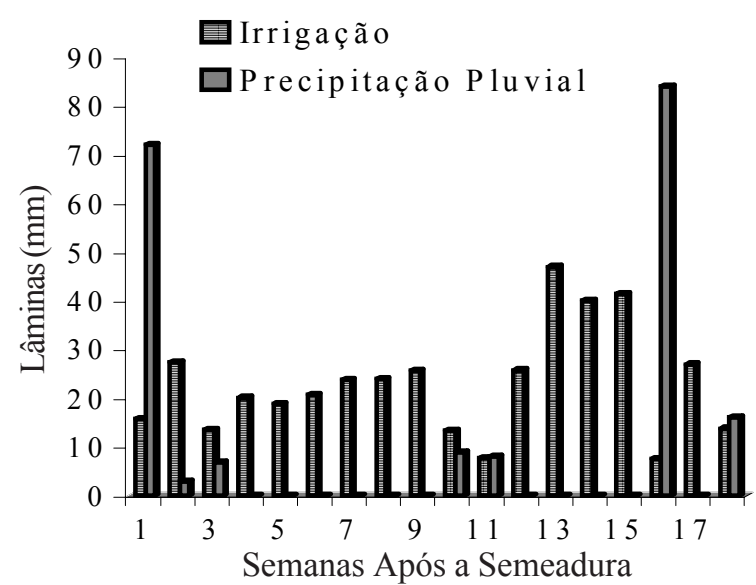

Figura 1. Lâminas de irrigação aplicadas e precipitação pluvial ocorrida no decorrer do experimento

A análise dos dados climáticos mostra a ocorrência de maiores precipitações nos períodos inicial e final do experimento. A radiação solar média diária foi de $271,8 \mathrm{~W} \mathrm{~m}^{-2}$. As temperaturas máxima e mínima oscilaram em torno de 23,7 e $14,0^{\circ} \mathrm{C}$, respectivamente. A umidade relativa do ar máxima variou entre 58 e 100\%, enquanto a mínima ficou entre 20 e $92 \%$. A velocidade do vento no período experimental foi moderada, com média de $2,2 \mathrm{~m} \mathrm{~s}^{-1}$.

$\mathrm{Na}$ Tabela 2 encontram-se os valores de coeficiente de cultura da cenoura nos diversos estádios de desenvolvimento, encontrados em diferentes fontes de literatura e determinados no presente estudo (FAO 56).

Neste trabalho foram obtidos valores de $\mathrm{K}_{\mathrm{c}}$ de 1,$15 ; 1,12$; 1,12 e 1,10, para os estádios de desenvolvimento inicial, crescimento rápido, intermediário e final, respectivamente. A metodologia proposta no Boletim FAO 56 leva em consideração o umedecimento da camada superficial do solo, afetando as estimativas do $\mathrm{K}_{\mathrm{c}}$ dos dois primeiros estádios, em comparação com os outros resultados baseados no Boletim FAO 24.
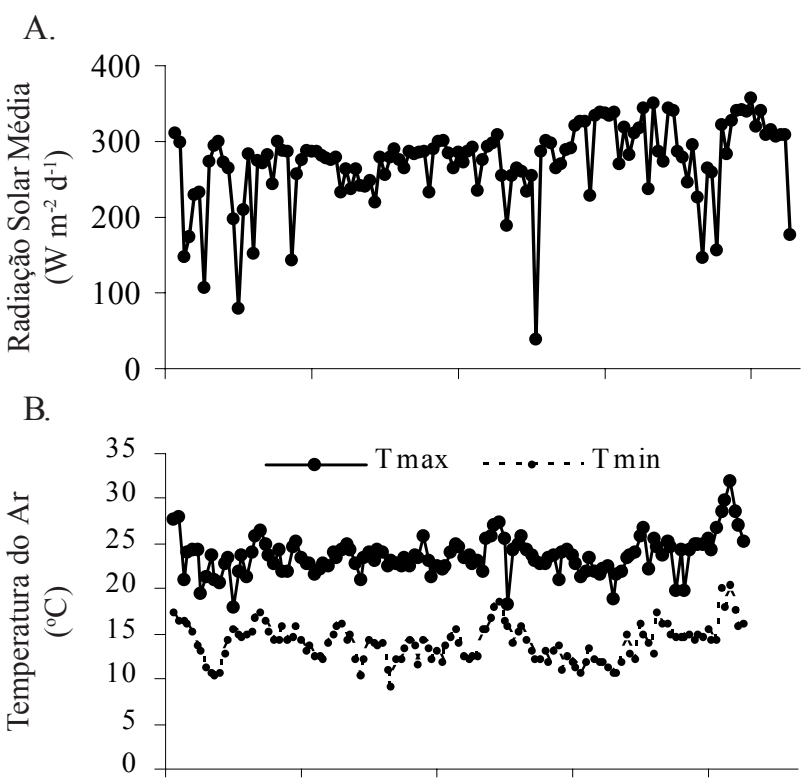

C.
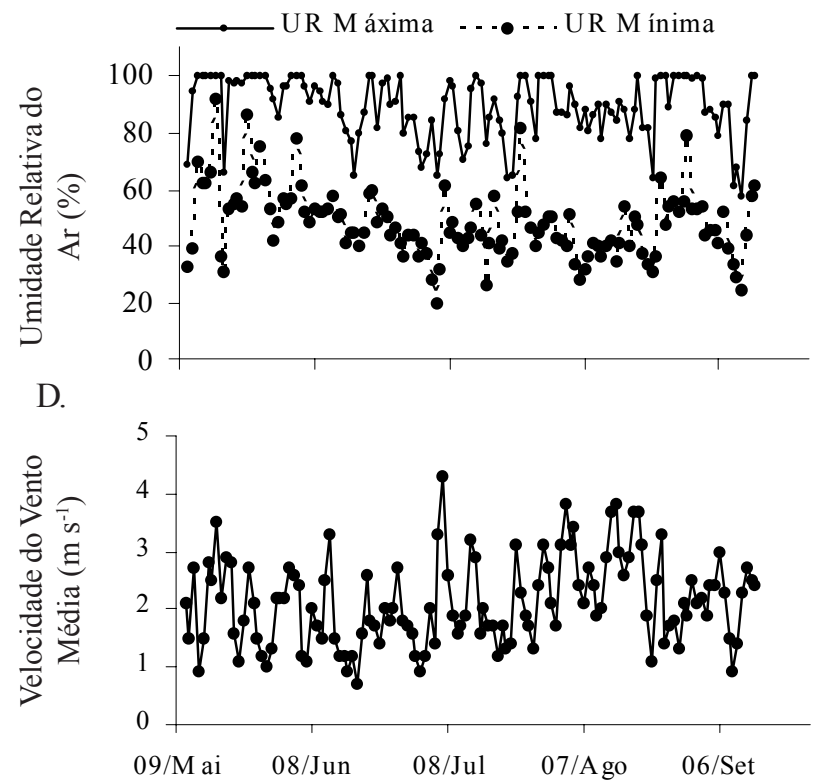

Figura 2. Radiação solar média diária (A), temperatura do ar máxima e mínima (B), umidade relativa do ar máxima e mínima (C) e velocidade do vento média diária (D)

Tabela 2. Valores de coeficiente de cultura $\left(\mathrm{K}_{\mathrm{c}}\right)$ da cenoura (Daucus carota L.) nos diversos estádios de desenvolvimento, obtidos com uso dos Boletins FAO 24 e FAO 56

\begin{tabular}{|c|c|c|c|c|c|}
\hline $\begin{array}{c}\text { Estádios de } \\
\text { Desenvolvimento }\end{array}$ & $\mathrm{K}_{\mathrm{c}}{ }^{1}$ & $\mathrm{~K}_{\mathrm{c}}{ }^{2}$ & $\mathrm{~K}_{\mathrm{c}}{ }^{3}$ & $\mathrm{~K}_{\mathrm{c}}^{4}$ & $\mathrm{~K}_{\mathrm{c}}{ }^{5}$ \\
\hline Inicial & - & 1,03 & 0,48 & $0,50-0,60$ & 1,15 \\
\hline Crescimento rápido & 0,77 & 0,80 & 0,77 & $0,70-0,85$ & 1,12 \\
\hline Intermediário & 0,99 & 1,16 & 1,47 & $1,00-1,15$ & 1,12 \\
\hline Final & 0,86 & 1,61 & 1,56 & $0,70-0,85$ & 1,10 \\
\hline $\begin{array}{l}\text { 1 Aragão Júnior et al. (1983) } \\
2 \text { Carvalho (1994) } \\
{ }^{3} \text { Giacóia Neto (1996) } \\
{ }^{4} \text { Silva \& Marouelli (FAO-24) } \\
\text { 5ilesente estudo (FAO-56) }\end{array}$ & & & & & \\
\hline
\end{tabular}

No transcorrer dos estádios inicial e de crescimento da cultura, o manejo da irrigação foi conduzido com turno de rega de 2 dias até o desbaste, efetuado aos 40 dias após o plantio. 
Assim, a superfície do solo permaneceu úmida e relativamente exposta nesse período, aumentando o valor do coeficiente $\mathrm{K}_{\mathrm{e}}$ e mantendo o $\mathrm{K}_{\mathrm{s}}$ igual à unidade; isto contribuiu para que o valor do coeficiente $\mathrm{K}_{c}$, determinado com aplicação da metodologia FAO 56, fosse maior que o $\mathrm{K}_{c}$ médio estimado de acordo com a metodologia recomendada no Boletim FAO 24 , como pode ser observado na Figura 3, na qual a curva $\mathrm{K}_{\mathrm{cb}}$ corresponde aos valores mínimos da evapotranspiração da cultura para condições adequadas de umidade do solo. As elevações bruscas que ocorrem na curva $\mathrm{K}_{\mathrm{cb}}+\mathrm{K}_{\mathrm{e}}$ correspondem aos períodos em que ocorreu irrigação ou chuva, umedecendo a superfície do solo e aumentando, temporariamente, o valor da evapotranspiração da cultura.

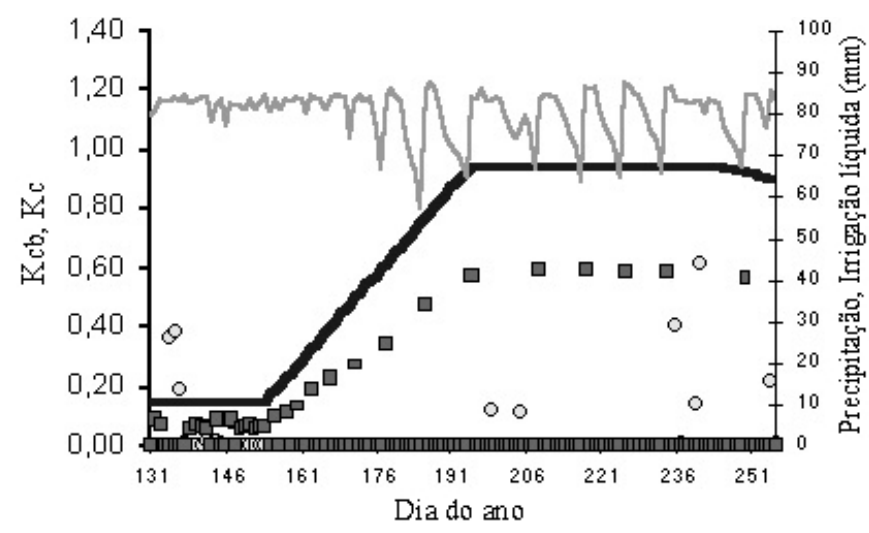

Kcb Kcb + Ke OPrecipitação a Irrigação líquida

Figura 3. Variação dos coeficientes $\mathrm{K}_{\mathrm{cb}} \mathrm{e} \mathrm{K}_{\mathrm{c}}\left(\mathrm{K}_{\mathrm{cb}}+\mathrm{K}_{\mathrm{e}}\right)$, precipitação pluvial e lâmina de irrigação, ao longo do ciclo da cultura da cenoura

Nos estádios intermediário e final, as irrigações foram feitas com menor freqüência e lâmina maior. Na região do Vale do Alto Paranaíba é usual efetuar-se a irrigação da cultura da cenoura, nesses estádios, com turno de rega máximo de 3 dias, quando se usa sistema pivô central. Embora a superfície do solo tenha ficado umedecida, a evaporação foi atenuada pelo sombreamento.

\section{CONCLUSÕES}

1. Os valores de $\mathrm{K}_{\mathrm{c}}$ para a cultura da cenoura explorada na região do Alto Paranaíba, MG, foram iguais a 1,15; 1,12; 1,12 e 1,10 para os estádios de desenvolvimento inicial, crescimento da cultura, intermediário e final, respectivamente, obtidos por meio da aplicação da metodologia proposta no Paper FAO 56.

2. Nos dois primeiros estádios, inicial e crescimento da cultura, os valores de $\mathrm{K}_{\mathrm{c}}$ foram expressivamente maiores que os obtidos com a metodologia FAO 24.

\section{LITERATURA CITADA}

Allen, R.G.; Pereira, L.S.; Raes, D.; Smith, M. Crop evapotranspiration - Guidelines for computing crop water requirements. Rome: FAO, 1998. 319p. FAO Irrigation and Drainage. Paper 56.

Aragão Júnior, T.C. Determinação do coeficiente de cultura (Kc) para a cenoura (Daucus carota L.) pelo método do balanço hídrico. Fortaleza, CE: UFC, 1983.45p. Dissertação Mestrado

Carvalho, J. de A. Coeficiente de cultura, avaliação econômica da produção e análise do crescimento da cenoura (Daucus carota L.) irrigada. Viçosa, MG: UFV, 1994. 78p. Tese Doutorado

Comissão de Fertilidade do Solo do Estado de Minas Gerais. Recomendação do uso de corretivos e fertilizantes no estado de Minas Gerais, $5^{\text {a }}$ aproximação. Viçosa, MG, 1999.359p.

Doorenbos, J.; Kassam, A.H. Yield response to water. Rome: FAO, 1979. 193p. FAO Irrigation and Drainage, Paper 33

Doorenbos J.; Pruitt, W.O. Guidelines for predicting crop water requirements, Rome: FAO, 1977. 179p. Irrigation and Drainage, Paper 24

Giacoia Neto, J. Efeito da profundidade do lençol freático na evapotranspiração e na produtividade da cultura de cenoura (Daucus carota L.). Viçosa, MG: UFV, 1996. 56p. Dissertação Mestrado

Pereira, L.S.; Allen, R.G. Novas aproximações aos coeficientes culturais. Engenharia Agrícola, Jaboticabal, v.16, n.4, p.118143, 1997.

Silva, W.L.C.; Marouelli, W.A. Manejo da irrigação em hortaliças no campo e em ambientes protegidos. In: Congresso Brasileiro de Engenharia Agrícola, 27, 1998, Poços de Caldas, MG. Manejo de irrigação. Poços de Caldas: SBEA-UFLA, 1998. p.311-348. 